\title{
PhoPQ two-component regulatory system plays a global regulatory role in antibiotic susceptibility, physiology, stress adaptation, and virulence in Stenotrophomonas maltophilia
}

Hsu-Feng Lu ${ }^{1,2+}$, Bo-Kuan Wu ${ }^{3+}$, Yi-Wei Huang ${ }^{3}$, Ming-Zhe Lee ${ }^{1}$, Ming-Fang Li ${ }^{4}$, Hsu-Jung Ho ${ }^{4}$, Hung-Chi Yang ${ }^{4^{*}}$ and Tsuey-Ching Yang ${ }^{3 *}$

\begin{abstract}
Background: Stenotrophomonas maltophilia, an opportunistic pathogen, is ubiquitously present in various environments, signifying its high capability of environmental adaptation. Two-component regulatory system (TCS) is a powerful implement to help organisms to survive in different environments. In clinic, treatment of S. maltophilia infection is difficult because it is naturally resistant to many antibiotics, highlighting the necessity to develop novel drugs or adjuvants. Given their critical and extensively regulatory role, TCS system has been proposed as a convincing target for novel drugs or adjuvants. PhoPQ TCS, a highly conserved TCS in several pathogens, plays crucial roles in lowmagnesium adaption, polymyxin resistance, and virulence. In this study, we aimed to characterize the role of PhoPQ TCS of S. maltophilia in antibiotic susceptibility, physiology, stress adaptation, and virulence.

(Continued on next page)
\end{abstract}

\footnotetext{
*Correspondence: hcyang@mail.ypu.edu.tw; tcyang@ym.edu.tw

${ }^{\dagger}$ Hsu-Feng Lu and Bo-Kuan Wu contributed equally to this work.

${ }^{4}$ Department of Medical Laboratory Science and Biotechnology, Yuanpei University of Medical Technology, Hsinchu, Taiwan

${ }^{3}$ Department of Biotechnology and Laboratory Science in Medicine, National Yang-Ming University, Taipei, Taiwan

Full list of author information is available at the end of the article
}

(C) The Author(s). 2020 Open Access This article is licensed under a Creative Commons Attribution 4.0 International License, which permits use, sharing, adaptation, distribution and reproduction in any medium or format, as long as you give appropriate credit to the original author(s) and the source, provide a link to the Creative Commons licence, and indicate if changes were made. The images or other third party material in this article are included in the article's Creative Commons licence, unless indicated otherwise in a credit line to the material. If material is not included in the article's Creative Commons licence and your intended use is not permitted by statutory regulation or exceeds the permitted use, you will need to obtain permission directly from the copyright holder. To view a copy of this licence, visit http://creativecommons.org/licenses/by/4.0/ The Creative Commons Public Domain Dedication waiver (http://creativecommons.org/publicdomain/zero/1.0/) applies to the data made available in this article, unless otherwise stated in a credit line to the data. 


\begin{abstract}
(Continued from previous page)
Results: To characterize PhoPQ system, phoP single mutant as well as phoP and phoQ double mutant were constructed. Distinct from most phoPQ systems of other microorganisms, two features were observed during the construction of phoP and phoQ single deletion mutant. Firstly, the phoQ mutant was not successfully obtained. Secondly, the compromised phenotypes of phoP mutant were not reverted by complementing an intact phoP gene, but were partially restored by complementing a phoPQ operon. Thus, wild-type KJ, phoP mutant (KU $\triangle \mathrm{PhoP}$ ), phoPQ mutant (KJPhoPQ), and complemented strain (KJPPhoPQ (pPhoPQ)) were used for functional assays, including antibiotic susceptibility, physiology (swimming motility and secreted protease activity), stress adaptation (oxidative, envelope, and iron-depletion stresses), and virulence to Caenorhabditis elegans. $\mathrm{K} \Delta \Delta \mathrm{PhoPQ}$ totally lost swimming motility, had enhanced secreted protease activity, increased susceptibility to antibiotics ( $\beta$-lactam, quinolone, aminoglycoside, macrolide, chloramphenicol, and sulfamethoxazole/ trimethoprim), menadione, $\mathrm{H}_{2} \mathrm{O}_{2}$, $\mathrm{SDS}$, and 2,2'dipyridyl, as well as attenuated virulence to C. elegans. Trans-complementation of KJAPhoPQ with phoPQ reverted these altered phenotypes to the wild-type levels.
\end{abstract}

Conclusions: Given the critical and global roles of PhoPQ TCS in antibiotic susceptibility, physiology, stress adaptation, and virulence, PhoPQ is a potential target for the design of drugs or adjuvants.

Keywords: Stenotrophomonas maltophilia, Swimming, Oxidative stress, Antibiotic resistance, Virulence

\section{Background}

Bacterial survival relies on their ability to cope with the challenge of environmental pressures. The two-component regulatory system (TCS) is a multi-variable regulatory mechanism, which is widely present in bacteria and contributes to the adaptation of bacteria to environmental stress. In a typical prokaryotic TCS, an environmental stimulus is sensed by the sensor kinase (SK), leading to its autophosphorylation, and transfer of the phosphoryl group of SK to the cognate response regulator (RR). The activated RR binds to the promoters of the target genes, ultimately leading to coordinated changes in global gene expression profiles [1,2].

The PhoP/PhoQ (PhoPQ) TCS system is highly conserved among many pathogenic or non-pathogenic gram-negative bacteria [3]. The PhoPQ TCS consists of the SK PhoQ and the RR PhoP. PhoQ can respond to several environmental signals by autophosphorylation, including low- $\mathrm{Mg}^{2+}$ and low- $\mathrm{Ca}^{2+}$ conditions, acidic $\mathrm{pH}$, the presence of cationic antimicrobial peptides (CAP), and osmotic upshift [4-7]. Phosphorylated PhoQ transfers the phosphate to PhoP, and activated PhoP further regulates the expression of downstream genes (PhoP regulon). Although PhoQs in different microorganisms can sense the same or similar environmental signals, the regulons triggered by phosphorylated PhoP vary in different species of bacteria $[8,9]$. The PhoPQ systems of human pathogens have been shown to participate in low-magnesium adaptation, acid tolerance, polymyxin resistance, and virulence [10-12]. Given the extensive coverage of PhoP regulon, loss of function of PhoPQ generally has a pleiotropic impact on a bacterium [3].

Stenotrophomonas maltophilia is a gram-negative, ubiquitous environmental bacterium, which can be isolated from water, soils, plant roots, animals, and humans. It is equipped with a wide range of activities, including plant growth promotion, pollutants breakdown, secondary metabolites production, and human pathogenicity [13]. S. maltophilia is not a highly virulent pathogen, but it has emerged as an important opportunistic pathogen. Chronic infections by this organism are prevalent in immunocompromised or cystic fibrosis patients. S. maltophilia infections are difficult to treat, as it is intrinsically resistant to various antibiotics [14].

Given the notable diversity of its habitats, S. maltophilia harbors an array of systems to adapt to different environmental niches. Sequencing of the S. maltophilia genome revealed the presence of at least 43 TCS regulatory proteins [15], implying that the ability of S. maltophilia to adapt to different ecological niches is noteworthy and the underlying mechanisms are very complex. Despite the fact that the significance of PhoPQ TCS in other bacteria have been widely reported [3], the functions of PhoPQ TCS in S. maltophilia have not been thoroughly examined except a recent report by Liu's group [12]. Liu's group has demonstrated that PhoPQ TCS of S. maltophilia is involved in the resistance to aminoglycoside and polymyxin, as well as the membrane permeability [12]. Therefore, in this study we examined the role of PhoPQ TCS in antibiotic susceptibility, physiological functions, stress adaptation, and virulence to nematode.

\section{Results}

Role of phoPQ TCS in bacterial viability in magnesiumlimited condition

To study the functions of phoPQ in S. maltophilia, in frame deletions were created at the $p h o P$ and $p h o Q$ loci, either individually or in combination, by double crossover homologous recombination, yielding $\mathrm{KJ} \Delta \mathrm{PhoP}$ and $\mathrm{KJ} \Delta \mathrm{PhoPQ}$. Despite several attempts, a phoQ mutant was not constructed. 
In S. maltophilia and in numerous other bacteria, the PhoPQ TCS is activated by limiting the availability of magnesium and the phoP mutant shows growth impairment in this condition $[10,12]$. We confirmed impairment of the growth of $\mathrm{KJ} \Delta \mathrm{PhoP}$ and $\mathrm{KJ} \Delta \mathrm{PhoPQ}$ on minimal XOLNG medium without $\mathrm{MgCl}_{2}$. The complementation of $\mathrm{KJ} \Delta \mathrm{PhoP}$ with plasmid pPhoPQ partially restored the growth defect of $\mathrm{KJ} \Delta \mathrm{PhoP}$ but $\mathrm{pPhoPQ}$ complementation in $\mathrm{KJ} \Delta \mathrm{PhoPQ}$ fully restored its growth. However, complementation of $\mathrm{KJ} \Delta \mathrm{PhoP}$ with plasmid pPhoP did not restore the growth defect (Fig. 1).

To explain the failure of phoP complementation assay in $\mathrm{KJ} \Delta \mathrm{PhoP}$, phoP expression in the KJ $\Delta \mathrm{PhoP}$ (pPhoP) was evaluated. While the phoP transcript was expressed in $\mathrm{KJ} \Delta \mathrm{PhoP}$ (pPhoP) and KJ $\Delta \mathrm{PhoP}$ (pPhoPQ) (Fig. 2a), only marginal to moderate levels of PhoP protein were detected in both strains (Fig. 2b; Fig. S1). Similar assessment was also performed in $\mathrm{KJ} \Delta \mathrm{PhoPQ}$ by complementation of plasmids pPhoP, $\mathrm{pPhoQ}$, and $\mathrm{pPhoPQ}$, respectively. The same trend was observed that abundant phoP transcript, but not PhoP protein, was detected in $\mathrm{KJ} \Delta \mathrm{PhoPQ}$ (pPhoP); however, both phoP transcript and PhoP protein reached significant levels in $\mathrm{KJ} \Delta \mathrm{PhoPQ}$ (pPhoPQ) (Fig. 2a $\& \mathrm{~b})$. This provided a reasonable explanation for the failure of phoP complementation in KJ $\Delta$ PhoP. It was worthily mentioned that there is a slight difference of migration of the PhoP proteins in the different lanes shown in Fig. $2 \mathrm{~b}$. This might be related to the phosphorylation status of PhoP proteins. The underlying mechanism for the absence of PhoP proteins in $\mathrm{KJ} \Delta \mathrm{PhoP}$ (pPhoP) and $\mathrm{KJ} \Delta \mathrm{PhoPQ}$ (pPhoP) is not immediately clear at present. Thus, the phoP complementation strains were not included in the following study.

\section{Role of PhoPQ TCS in antibiotic susceptibility}

The susceptibility of phoP or phoPQ mutants to antibiotics ceftazidime, ticarcillin/clavulanic acid, ciprofloxacin, levofloxacin, nalidixic acid, kanamycin, tobramycin, erythromycin, leucomycin, chloramphenicol, and trimethoprim-sulfamethoxazole (SXT) was examined. PhoP or PhoPQ deletion reduced the MICs by 4- to 64fold in all the antibiotics tested. Trans-complementation of $\mathrm{KJ} \Delta \mathrm{PhoPQ}$ with $\mathrm{pPhoPQ}$ restored resistance to all tested antibiotics (Table 1).

\section{Role of PhoPQ TCS in bacterial physiology}

The effects of the inactivation of phoP or phoPQ on bacterial physiology were assessed with respect to bacterial growth, swimming, and secreted protease activity. Bacterial growth was evaluated by monitoring the $\mathrm{OD}_{450 \mathrm{~nm}}$ at different growth time points. $\mathrm{KJ} \Delta \mathrm{PhoP}$ and $\mathrm{KJ} \Delta \mathrm{PhoPQ}$ had reduced, but not statistically significant, growth compared to wild-type KJ (Fig. 3a).

Next, the swimming motilities of KJ $\Delta$ PhoP and KJ $\Delta$ PhoPQ were evaluated. Inactivation of phoP or phoPQ of S. maltophilia KJ completely abolished the swimming motility. Complementation of $\mathrm{KJ} \triangle \mathrm{PQ}$ with $\mathrm{pPhoPQ}$ restored swimming motility to the wild-type level (Fig. 3b \& Fig. S2).

The secreted protease activities of $\mathrm{KJ}, \mathrm{KJ} \Delta \mathrm{PhoP}$, and $\mathrm{KJ} \Delta \mathrm{PhoPQ}$ were determined. Inactivation of phoP or phoPQ of strain $\mathrm{KJ}$ resulted in higher secreted protease activity and pPhoPQ complementation restored the secreted protease activity to the wild-type level (Fig. 3c \& Fig. S3).

\section{Role of PhoPQ TCS in stress adaptation}

The contribution of $p h o P$ or $p h o P Q$ toward adaptation to oxidative, envelope, and iron-depleted stresses was investigated.

The role of phoP or phoPQ in oxidative stress response was tested by $\mathrm{H}_{2} \mathrm{O}_{2}$ and menadione (MD) susceptibility assays. $\mathrm{KJ} \Delta \mathrm{PhoP}$ and $\mathrm{KJ} \Delta \mathrm{PhoPQ}$ were more susceptible to $\mathrm{H}_{2} \mathrm{O}_{2}$ and $\mathrm{MD}$ than wild-type $\mathrm{KJ}$. The $\mathrm{H}_{2} \mathrm{O}_{2}$ and $\mathrm{MD}$ tolerances of $\mathrm{KJ} \Delta \mathrm{PhoPQ}$ were reverted by complementing with the pPhoPQ plasmid (Fig. 4a \& b).

The tolerances to envelope stress and iron depletion stress were assessed by examining cell viability in sodium

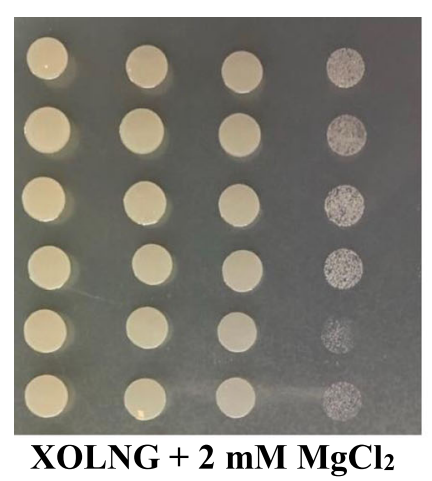

\section{KJ(pRK415)}

KJ $\Delta$ PhoP(pRK415)

KJ $\Delta$ PhoP(pPhoP)

KJ $\Delta$ PhoP(pPhoPQ)

KJ $\triangle \mathrm{PhoPQ}(\mathrm{pRK415)}$

KJ $\Delta$ PhoPO(pPhoPQ)

XOLNG + 2 mM MgCl 2

Fig. 1 Impact of phoPQ TCS on bacterial growth in magnesium limited condition. The logarithmic-phase bacterial cells of $2 \times 10^{5} \mathrm{CFU} / \mu \mathrm{l}$ were $10-$ fold serially diluted. Bacterial suspension $(5 \mu \mathrm{l})$ was spotted onto the minimal medium XOLNG with or without $\mathrm{MgCl}_{2}$. After $24 \mathrm{~h}$ of incubation at $37^{\circ} \mathrm{C}$, the growth of bacterial cells was observed 
(A)

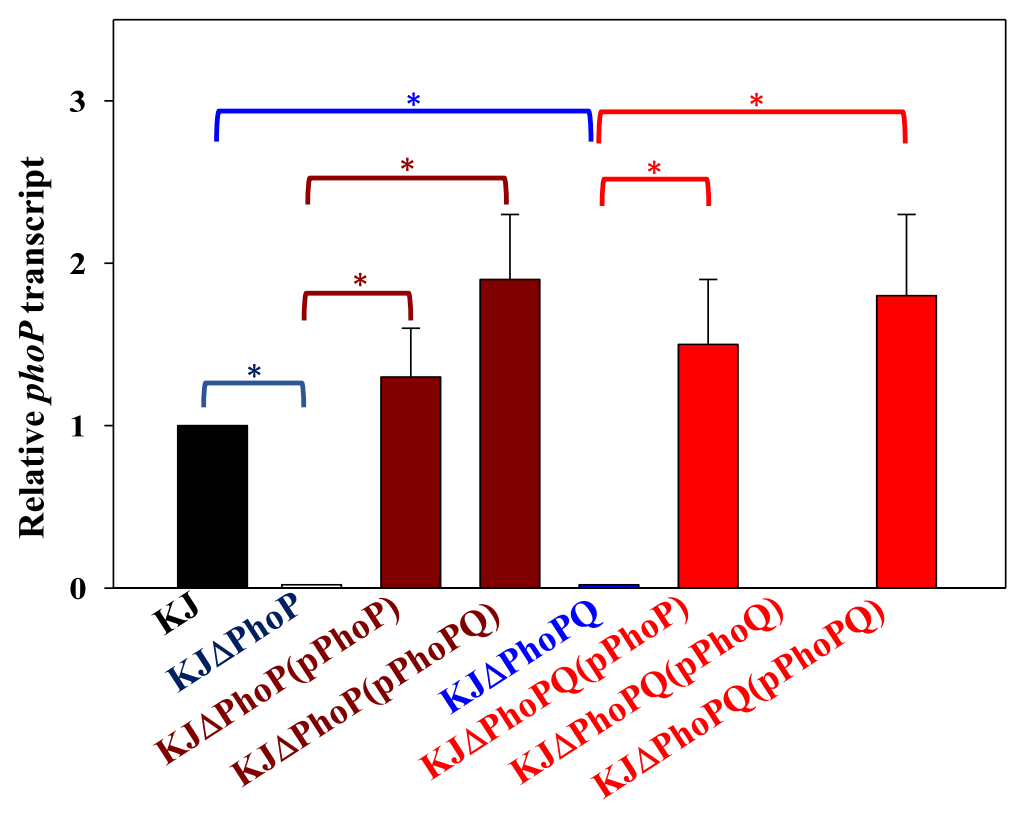

(B)

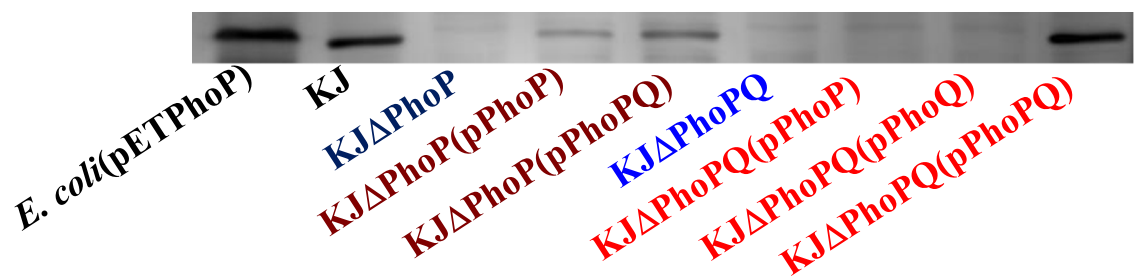

Fig. 2 Expression of PhoP transcript and PhoP protein in wild-type K, its derived mutants, and the complementary strains. a PhoP transcript levels. Overnight culture of bacterial cells was inoculated into fresh $L B$ with an initial $O_{450 n m}$ of 0.15 . Cells were grown aerobically for $5 \mathrm{~h}$ before measuring phoP transcript using qRT-PCR. Bars represent the average values from three independent experiments. Error bars represent the standard error of the mean. *, $P<0.01$, significance calculated by Student's $t$ test. b PhoP protein levels. Whole bacterial cell lysates were denatured and separated by $14 \%$ SDS-PAGE and then transferred onto polyvinylidene difluoride (PVDF) membranes. The membrane was incubated with anti-PhoP antibodies and secondary mouse anti-rabbit lgG immunoglobulin conjugated to horseradish peroxidase. The reaction was developed with a mixture of diamino benzidine and 30\% hydrogen peroxide. Full-length blots/gels are presented in Supplementary's Figure S1

dodecyl sulfate (SDS)-containing and 2,2'-dipyridyl (DIP)-containing LB agars, respectively. The viabilities of $\mathrm{KJ} \Delta \mathrm{PhoP}$ and $\mathrm{KJ} \Delta \mathrm{PhoPQ}$ were apparently compromised compared to that of wild-KJ. Complementation of $\mathrm{KJ} \Delta \mathrm{PhoPQ}$ with $\mathrm{pPhoPQ}$ restored cell viability (Fig. 4c \& d).

\section{Role of PhoPQ TCS in virulence to nematode}

The relationship between PhoPQ TCS and virulence was assessed using the Caenorhabditis elegans model. Median survivals for KJ (pRK415), KJ $\Delta$ PhoPQ (pRK415), and $\mathrm{KJ} \Delta \mathrm{PhoPQ}$ (PhoPQ) were 17, 22.5, and 11 days, respectively (Fig. 5). KJ $\Delta \mathrm{PhoPQ}$ was less virulent to C. elegans than wild-type KJ, and trans-complementation of
$\mathrm{KJ} \Delta \mathrm{PhoPQ}$ with plasmid $\mathrm{pPhoPQ}$ restored virulence to C. elegans.

\section{PhoPQ TCS regulates the MD-mediated upexpression of sodA1, katA2, and katE}

We were interested in elucidating the possible PhoPQregulated candidate genes involved in the above phenotypes. Of the phenotypes assayed, oxidative stress alleviation was selected for following study since the underlying mechanisms involved in MD-mediated oxidative stress alleviation of $S$. maltophilia are extensively surveyed in our previous studies [16-21]. The known mechanisms against oxidative stress in S. maltophilia include superoxide dismutases (SodA1, SodA2, SodB, and SodC1C2) [16], catalases (KatA1, KatA2, KatE, and KatMn), alkyl hydroperoxide 
Table 1 Antibiotic susceptibilities of S. maltophilia $\mathrm{KJ}, \mathrm{KJ} \Delta \mathrm{PhoPQ}$, and $\mathrm{K} \Delta \mathrm{PhoPQ}$ (pPhoPQ)

\begin{tabular}{|c|c|c|c|c|}
\hline \multirow[t]{2}{*}{ Antibiotic } & \multicolumn{4}{|c|}{ MIC $(\mu \mathrm{g} / \mathrm{ml})$} \\
\hline & $\begin{array}{l}\text { KJ } \\
\text { (pRK415) }\end{array}$ & KJ $\Delta$ PhoP (pRK415) & $\mathrm{KJ} \Delta \mathrm{PhoPQ}$ (pRK415) & $\mathrm{KJ} \Delta \mathrm{PhoPQ}(\mathrm{pPhoPQ})$ \\
\hline \multicolumn{5}{|l|}{$\beta$-lactam } \\
\hline Ceftazidime & 256 & 64 & 16 & 256 \\
\hline Ticarcillin-clavulanate & 128 & 16 & 8 & 128 \\
\hline \multicolumn{5}{|l|}{ Quinolone } \\
\hline Ciprofloxacin & 1 & 0.25 & 0.125 & 1 \\
\hline Levofloxacin & 1 & 0.125 & 0.125 & 1 \\
\hline Nalidixic acid & 8 & 2 & 2 & 8 \\
\hline \multicolumn{5}{|l|}{ Aminoglycoside } \\
\hline Kanamycin & 256 & 4 & 4 & 128 \\
\hline Tobramycin & 256 & 4 & 4 & 128 \\
\hline \multicolumn{5}{|l|}{ Macrolide } \\
\hline Erythromycin & 64 & 16 & 8 & 64 \\
\hline Leucomycin & 256 & 32 & 32 & 256 \\
\hline Chloramphenicol & 8 & 2 & 2 & 8 \\
\hline Sulfamethoxazole/trimethoprim & 2 & 0.5 & 0.25 & 2 \\
\hline
\end{tabular}

reductase (AhpC) [17], efflux pumps (MacABCsm, SmeYZ, and SmeVWX) [18-20], and the formaldehyde detoxification system FadACB [21]. KJ and KJ $\triangle \mathrm{PhoPQ}$ were cultured in the LB broth without or with MD, and the expressions of these oxidative stress-associated genes were assessed by qRT-PCR. Of thirteen genes surveyed, six genes $(\operatorname{sod} A 1$, katA2, katE, ahpC, smeV, and $f a d A$ ) in wild-type KJ were upregulated upon MD challenge (Fig. S4). The expressions of the six upregulated genes in $\mathrm{KJ} \Delta \mathrm{PhoPQ}$ with or without $\mathrm{MD}$ treatment were further assessed. In the absence of $\mathrm{MD}$, the six transcripts in $\mathrm{KJ}$ and $\mathrm{KJ} \Delta \mathrm{PhoPQ}$ were comparable. Compared to those in MD-treated KJ cells, the $\operatorname{sodA1}$, $k a t A 2$, and $k a t E$ transcript levels were apparently dropped in MD-treated $\mathrm{KJ} \Delta \mathrm{PhoPQ}$ cells (Fig. 6).

\section{Discussion}

PhoPQ is a highly conserved TCS in several gramnegative microbes. Compared to several known PhoPQ TCSs of other gram-negative human pathogens, the PhoPQ TCS of S. maltophilia has some special features, as revealed in this study. (i) The phoQ mutant cannot be obtained. (ii) The complementary plasmid pPhoP can be successfully transcribed in $\mathrm{KJ} \Delta \mathrm{PhoP}$, but PhoP protein is hardly detected in significant levels (Fig. 2). (iii) Compared to known PhoPQ systems, the functions of PhoPQ system of $S$. maltophilia are the most comprehensive, contributing to antibiotic susceptibility, physiology, stress adaptation, and virulence.

The unavailability of the phoQ mutant in this study was reminiscent of the essentiality of PhoPQ in Xanthomonas campestris [22]. With the exemption of Xanthomonas campestris, the reported phoQ orthologs in other bacteria, such as Escherichia coli, Salmonella enterica, Pseudomonas aeruginosa, and P. fluorescens, are mutable [8, 23, 24 . Peng et al. have proposed the polygenic transcriptional rewiring model to explain the essentiality of PhoPQ in $X$. campestris. They suggested that the PhoP regulon of $X$. campestris includes several essential genes, but the PhoP regulons of other bacteria do not [22]. In contrast to PhoP essentiality in $X$. campestris, the phoP deletion mutant is viable in $S$. maltophilia. Thus, we proposed two possibilities for explanation. (i) PhoQ may interact with other response regulator(s) to globally activate several regulatory circuits, in addition to its original cognate PhoP. In several gram-negative bacteria, it is common to have cross-talk between noncognate pairs of sensor kinase and response regulator. The PhoQ-associated regulons may include several genes essential for the viability of $S$. maltophilia. (ii) Non-phosporylated PhoP may have a toxic effect and inhibit growth.

A peculiar observation, which has not been reported in other species, is that, in contrast to $\mathrm{pPhoPQ}$, the plasmid pPhoP cannot successfully complement the compromised phenotype of KJ $\Delta$ PhoP. Similar experiment design has been observed by Liu et al., who complemented a phoP mutant of $S$. maltophilia S22 with a phoPQ-containing plasmid, but not with a phoP-containing plasmid [12]. They demonstrated that phoP inactivation causes a polar effect on the expression of downstream phoQ gene [12]. In the present study, we verified that phoP transcript, but not sufficient PhoP protein, was detectable in KJ $\Delta \mathrm{PhoP}$ (pPhoP) and KJ $\Delta$ PhoPQ (pPhoP), while both the phoP 
(A)

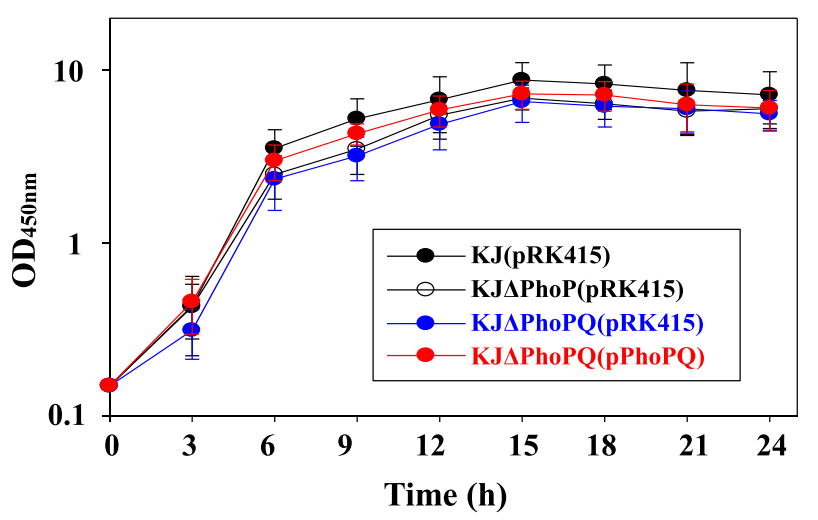

(B)

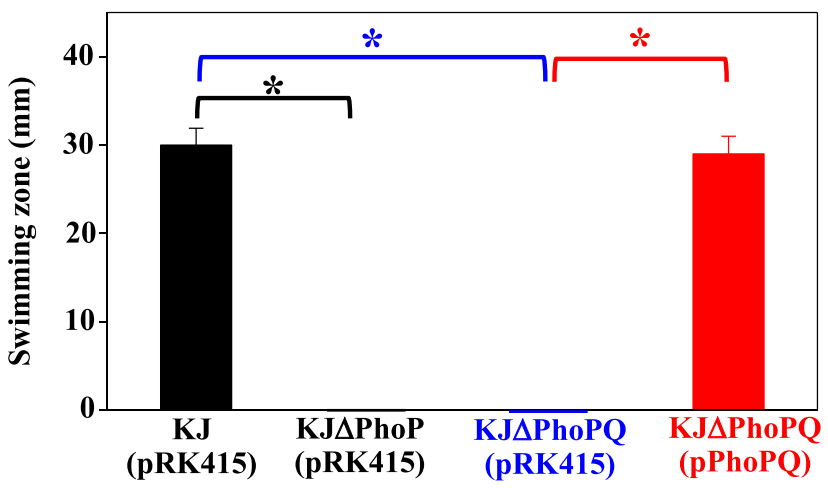

(C)

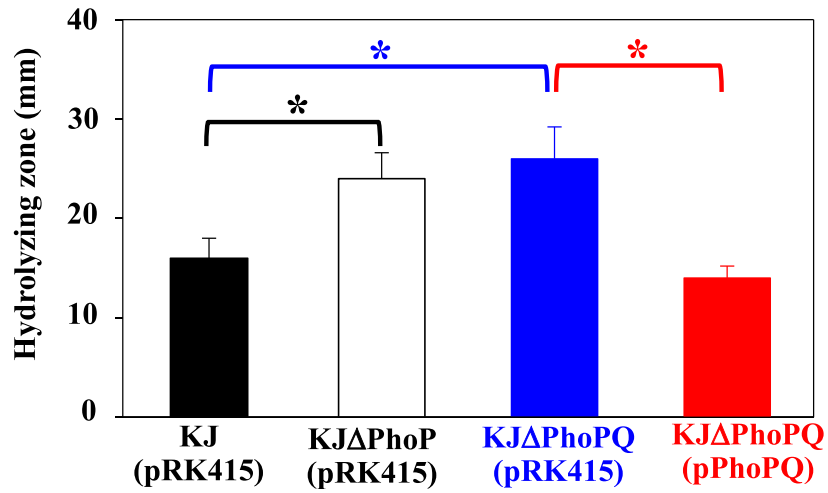

Fig. 3 Roles of phoPQ TCS in physiological functions. a Roles of phoPQ TCS in bacterial growth. Overnight culture of bacterial cells was inoculated into fresh $L B$ broth with an initial $O_{4} D_{40 n m}$ of 0.15 . The bacterial growth was monitored by recording the $\mathrm{OD}_{450 \mathrm{~nm}}$ every $3 \mathrm{~h}$. Error bars represent the standard error of the mean. ${ }^{*}, P<0.01$, significance calculated by Student's $t$ test. $\mathbf{b}$ Roles of phoPQ TCS in swimming motility. The logarithmic-phase bacterial culture was adjusted to $\mathrm{OD}_{450 \mathrm{~nm}}$ of 1.0. Five-microliter suspension was inoculated into the swimming agar (1\% tryptone, $0.5 \% \mathrm{NaCl}$, and $0.15 \%$ agar) and the swimming zones were recorded after $48-\mathrm{h}$ incubation at $37^{\circ} \mathrm{C}$. Bars represent the average values from three independent experiments. Error bars represent the standard error of the mean. ${ }^{*}, P<0.01$, significance calculated by Student's $t$ test. $\mathbf{c}$ Roles of phoPQ TCS in secreted protease activity. The logarithmic-phase bacterial culture was adjusted to $\mathrm{OD}_{450 \mathrm{~nm}}$ of 1.0 and $40 \mu \mathrm{l}$ bacterial aliquot were inoculated into the skim milk agar plate. The proteolytic activity of bacteria was assessed by measuring the transparent zones around the bacteria after incubation for $72 \mathrm{~h}$ at $37^{\circ} \mathrm{C}$. Bars represent the average values from three independent experiments. Error bars represent the standard error of the mean. ${ }^{*}, P<0.01$, significance calculated by Student's $t$ test 
(A)

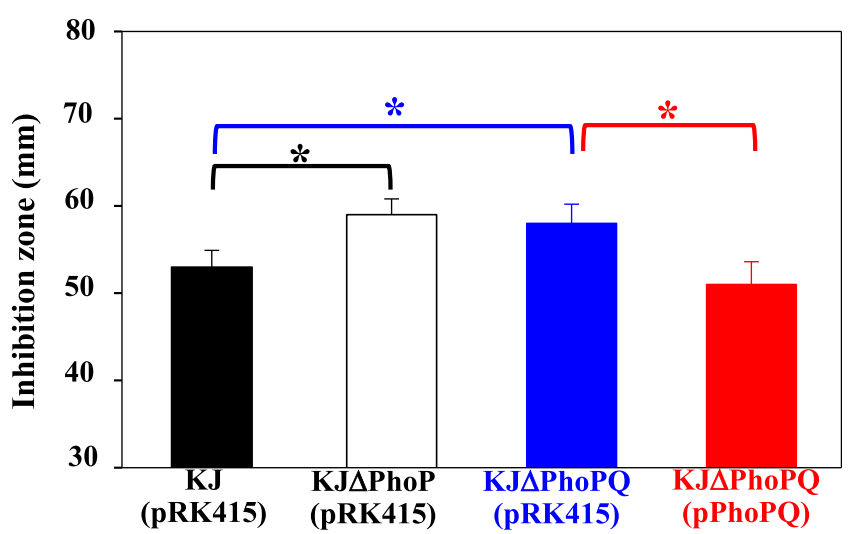

(B)

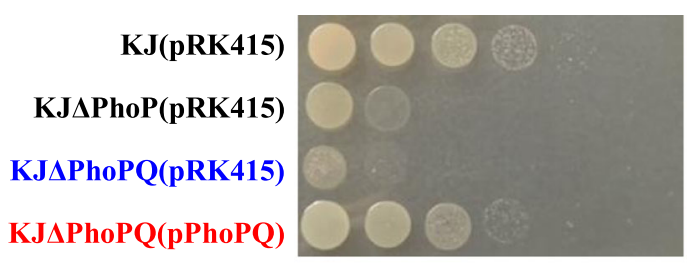

(C)

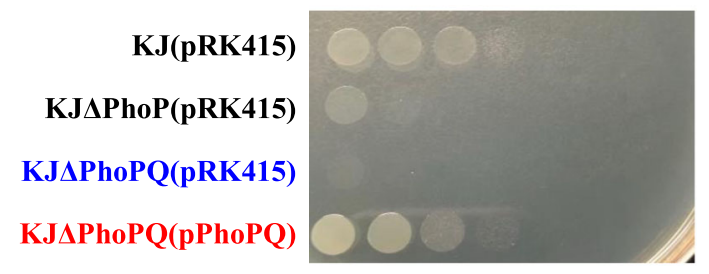

(D)

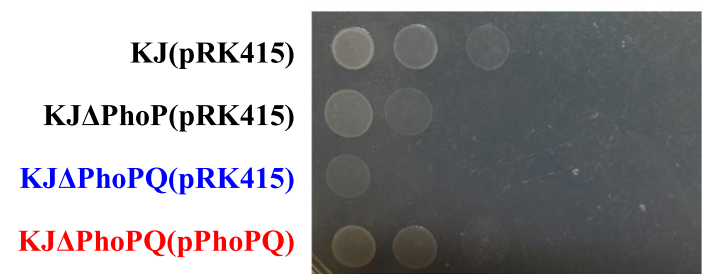

Fig. 4 Roles of phoPQ TCS in stress adaptation. a Roles of phoPQ TCS in $\mathrm{H}_{2} \mathrm{O}_{2}$ susceptibility. LB agar was uniformly spread with the bacterial cell suspension tested. Sterile filter paper soaked in $15 \mu \mathrm{l}$ of $20 \% \mathrm{H}_{2} \mathrm{O}_{2}$ was placed onto the center of agar. The diameter of growth inhibition zone was measured after $24-\mathrm{h}$ incubation at $37^{\circ} \mathrm{C}$. Bars represent the average values from three independent experiments. Error bars represent the standard error of the mean. ${ }^{*}, P<0.01$, significance calculated by Student's $t$ test. $\mathbf{b}$ Roles of phoPQ TCS in MD susceptibility. The logarithmicphase bacterial culture was adjusted to an $\mathrm{OD}_{450 \mathrm{~nm}}$ of 1.0 and serially diluted 10-fold. Five-microliter suspensions were applied onto the LB agar containing $50 \mathrm{\mu g} / \mathrm{ml} \mathrm{MD}$. After $24-\mathrm{h}$ incubation at $37^{\circ} \mathrm{C}$, cell viability was imaged. c Roles of phoPQ TCS in SDS susceptibility. The logarithmicphase bacterial culture was adjusted to an $\mathrm{OD}_{450 \mathrm{~nm}}$ of 1.0 and serially diluted 10 -fold. Five-microliter suspensions were applied onto the LB agar containing $0.01 \% \mathrm{SDS}$. After $24-\mathrm{h}$ incubation at $37^{\circ} \mathrm{C}$, cell viability was imaged. $\mathbf{d}$ Roles of phoPQ TCS in iron depletion tolerance. The logarithmicphase bacterial culture was adjusted to $\mathrm{OD}_{450 \mathrm{~nm}}$ of 1.0 and serially diluted 10 -fold. Five-microliter suspensions were applied onto the LB agar containing $45 \mu \mathrm{g} / \mathrm{ml}$ DIP. After $24-\mathrm{h}$ incubation at $37^{\circ} \mathrm{C}$, cell viability was imaged 


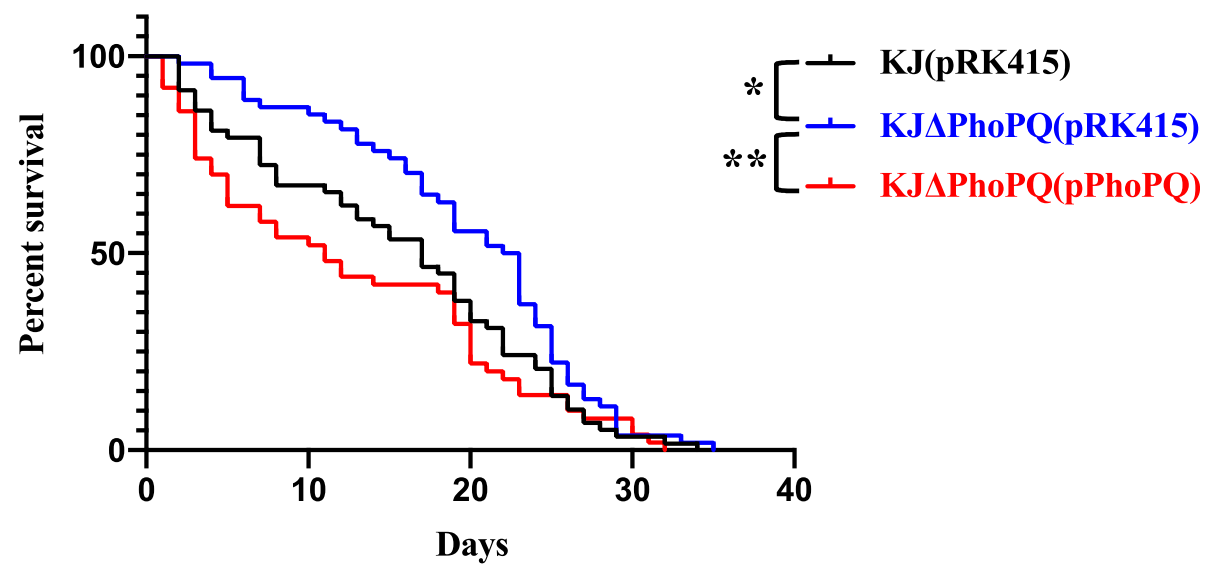

Fig. 5 Role of phoPQ in C. elegans survival. Sixty worms at the $L 4$ stage were transferred to S. maltophilia-seeded NGM agar plates and scored for survival daily

transcript and PhoP protein were detectable in $\mathrm{KJ} \Delta \mathrm{PhoPQ}$ (pPhoPQ) (Fig. 2). It seemed that an intact phoP-phoQ transcript is critical for a successful translation of the phoPQ transcript. Inactivation of $p h o P$ has a negative impact on the stability of $p h o Q$ transcript [12]. On the other hand, loss-offunction of $p h o Q$ significantly decreased the PhoP protein levels (Fig. 2b), implying the involvement of PhoQ in stability of PhoP protein. The complex interplay between the stability of $p h o P-p h o Q$ transcript and the functions of PhoP and PhoQ proteins is needed to be further elucidated.

The contribution of the PhoPQ system to antibiotic resistance has been reported in other gram-negative bacteria, but was limited to polymyxin and tetracycline $[1,23$,
25]. A notable observation of this study is that the PhoPQ of $S$. maltophilia had a marked impact on resistance to all the antibiotics tested (Table 1). Of all reported PhoPQ systems in gram-negative bacteria, the contribution of the $S$. maltophilia PhoPQ TCS to antibiotic resistance is the most comprehensive and prominent.

Enzymatic hydrolysis and efflux pump extrusion are two known oxidative stress alleviation systems for Gramnegative bacteria to deal with oxidative stress [26, 27]. In addition, a formaldehyde detoxification system, FadACB, has recently been reported to contribute to oxidative stress alleviation in S. maltophilia [21]. SoxR and OxyR are two well-known regulators involved in the regulatory

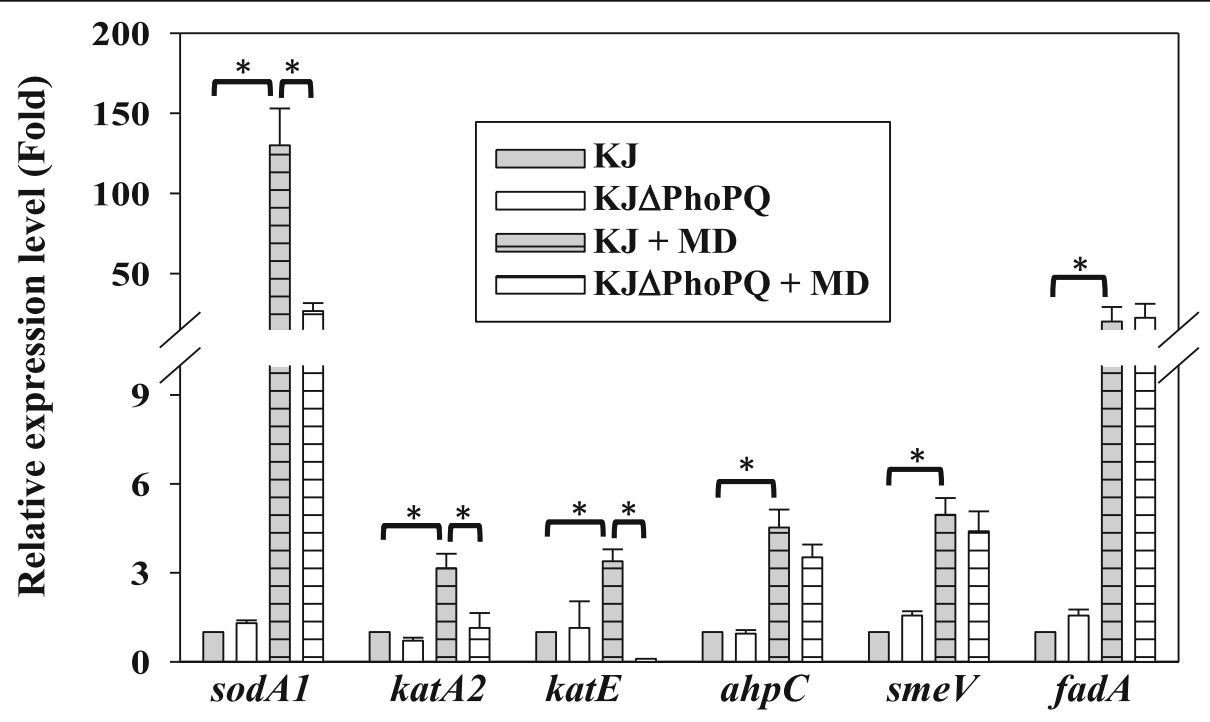

Fig. 6 The regulatory role of PhoPQ TCS in the expression of sodA1, katA2, katE, ahpC, smeV, and fadA genes. Overnight culture of KJ and KIPPhoPQ cells was inoculated into fresh $\mathrm{LB}$ broth without or with $\mathrm{MD}$ at an initial $\mathrm{OD}_{450 \mathrm{~nm}}$ of 0.15 . Cells were grown aerobically for $5 \mathrm{~h}$ before measuring sodA1, katA2, katE, ahpC, smeV, and fadA transcripts using qRT-PCR. All values were normalized to the transcript of MD-non-treated KJ cells. Bars represent the average values from three independent experiments. Error bars represent the standard error of the mean. ${ }^{*}, P<0.001$, significance calculated by Student's $t$ test 
circuits [28]. In this article, we further extended the understanding in the regulation of oxidative stress alleviation systems in S. maltophilia. PhoPQ TCS participates in the MD-mediated oxidative stress alleviation by upregulating the expression of $\operatorname{sodA1}$, katA2, and katE (Fig. 6). Since the consensus sequence for PhoP binding sites of S. maltophilia is not clear right now, we tried to survey whether the promoter regions of the three genes have conserved PhoP binding sites referenced from the reported PhoP boxes of $E$. coli and P. aeruginosa $[29,30]$ and on positive results were obtained.

For a successful colonization or infection, a bacterium should be able to invade host cells and have the functional capacity to defend itself from host-imposed stresses. Swimming motility is a critical ability for bacterial accessibility to host cells and the secreted protease activity is required to damage the host epithelial barrier and resist the action of host's virulence factors [31]. Innate/adaptive immunity and nutritional immunity are host defense mechanisms against pathogen invasion. ROS-mediated oxidative stress, cationic antimicrobial peptides-mediated envelope stress, magnesium depletion and iron depletion are common stresses imposed by host cells. The PhoPQ system of $S$. maltophilia governs swimming and adaption to oxidative stress, envelope stress, and iron depletion; thus, a functional PhoPQ system is critical for a successful infection.

\section{Conclusions}

The PhoPQ system of S. maltophilia has been demonstrated involvement in the resistance to polymyxin B, chloramphenicol, aminoglycoside, and ampicillin [12]. In this study, we further extended the understanding of PhoPQ function in the resistance to ceftazidime, ticarcillin-clavulanate, quinolone, and sulfamethoxazole/ trimethoprim, which are effective antibiotics commonly used for the treatment of S. maltophilia infection [14]. Furthermore, PhoPQ system also contributes to swimming, stress adaptation (oxidative stress, envelope stress, and iron depletion), and virulence to $C$. elegans. These findings signify the potential of PhoPQ as a target for drug design or adjuvant therapy.

\section{Methods}

\section{Bacterial strains, media, plasmids, and primers}

Table S1 lists the bacterial strains, plasmids, and primers used in this study. The XOLNG minimal medium was prepared as described previously [32].

\section{Construction of in-frame deletion mutants}

The DNA fragments flanking the deleted genes were amplified by PCR and subsequently cloned into pEX18Tc to generate the mutagenic plasmids for double cross-over homologous recombination. The primers sets for the construction of mutagenic plasmids were PhoPN-F/ PhoPN-R and PhoQN-F/PhoQN-R for $\mathrm{p} \Delta \mathrm{PhoP}$, PhoQNF/PhoQN-R and PhoQC-F/PhoQC-R for $\mathrm{p} \Delta \mathrm{PhoQ}$, and PhoPN-F/PhoPN-R and PhoQC-F/PhoQC-R for $\mathrm{p} \triangle \mathrm{PhoPQ}$ (Table S1). These of mutagenic plasmids were transferred into $S$. maltophilia by conjugation. The procedures for conjugation, transconjugants selection, and confirmation of mutants correctness were carried out as described previously [33].

\section{Construction of phoP, phoQ, and phoPQ complementary plasmids, $\mathrm{PPhoP}, \mathrm{pPhoQ}$, and $\mathrm{pPhoPQ}$}

To obtain expression plasmids for complementation experiments, phoP, $p h o Q$, and $p h o P-p h o Q$ genes were amplified by $\mathrm{PCR}$ from strain $\mathrm{KJ}$ and cloned into pRK415. The used primer sets were PhoP-F/PhoP-R for phoP gene, PhoQ-F/PhoQ-R for phoQ gene, and PhoPQF/PhoPQ-R for phoP-phoQ genes (Table S1).

\section{Quantitative reverse transcription-PCR (qRT-PCR)}

The DNA-free RNA of bacterial cells was extracted using Total RNA Extraction Kit Mini (ARROWTEC) and reverse transcribed to into cDNA as described previously [17]. qRT-PCR was carried out by ABI Prism 7000 Sequence Detection System (Applied Biosystems, Foster City, CA, United States). The primers used for qRT-PCR are listed in Table S1. The relative levels of expression were calculated by the threshold cycle $\Delta \Delta C t$ method [34] using the 16S rRNA as the control. Each assay was independently performed at least three times.

\section{Preparation of polyclonal anti-rabbit PhoP antibody}

The phoP gene of S. maltophilia KJ was obtained by PCR using the primers PhoPHis-F and PhoPHis-R (Table S1) and then inserted into the $E$. coli expression vector pET30b, yielding pETPhoP. The E. coli BL21(DE3) was transformed with plasmid pETPhoP. Overnight-cultured E. coli BL21(pETPhoP) was subcultured into fresh LB broth and grown to logarithmic growth phase, IPTG was added at final concentrations of $1 \mathrm{mM}$ for $3 \mathrm{~h}$. Bacterial cells were disrupted by sonicating and cell debris were removed by centrifugation. The Recombinant PhoP-6xHis proteins were obtained from clear lysate with Ni-NTA resin. Purity of PhoP-6xHis was determined by SDSPAGE. Antibodies against PhoP-6xHis protein were raised by immunization of New Zealand rabbits with the purified PhoP-6xHis protein.

\section{Western blotting analysis}

Whole bacterial cell lysates were denatured and separated by $14 \%$ SDS-PAGE and then transferred onto polyvinylidene difluoride (PVDF) membranes. The blot was reacted with anti-PhoP antibodies and secondary mouse 
anti-rabbit IgG immunoglobulin conjugated to horseradish peroxidase (Sigma Chemical Co., St. Louis, MO).

\section{Antibiotics susceptibility test}

Antimicrobial susceptibility test was performed using the twofold agar dilution method on Mueller-Hinton agar according to Clinical and Laboratory Standards Institute (CLSI) guidelines [35]. Bacterial growth was recorded after incubation at $37^{\circ} \mathrm{C}$ for $16-18 \mathrm{~h}$. The minimal inhibitory concentration (MIC) was the lowest concentration of the antibiotic that completely inhibited bacterial growth. Antibiotics were purchased from Sigma Chemical Co. Each assay was independently performed at least three times.

\section{Swimming motility}

Overnight culture was adjusted to $\mathrm{OD}_{450}$ of 1.0 and $5 \mu \mathrm{L}$ aliquot was inoculated onto the surface of swimming agar ( $1 \%$ tryptone, $0.5 \% \mathrm{NaCl}$, and $0.15 \%$ agar). Plates were incubated at $37^{\circ} \mathrm{C}$ for $48 \mathrm{~h}$ and the diameter $(\mathrm{mm})$ of the migration zone of bacteria was measured [19]. Each assay was independently performed at least three times.

\section{Secreted protease activity assay}

The $1 \%$ skin milk-supplemented LB agar was prepared for secreted protease activity assay, with a 6-mm-diameter hole in the center for the convenience of bacterial loading. Bacterial culture tested was adjusted to an OD450 of 1.0 and $40 \mu \mathrm{L}$ aliquot was dripped onto the hole of the skim milk agar plates. The proteolytic activity of bacteria was assessed by measuring the transparent zones around the bacteria after $72 \mathrm{~h}$-incubation at $37^{\circ} \mathrm{C}$ [19]. Each assay was independently performed at least three times.

Menadione (MD), sodium dodecyl sulfate (SDS), and 2,2'dipyridyl (DIP) susceptibility assay

The logarithmic-phase bacterial culture was adjusted to an OD450nm of 1 and subsequently 10-fold serially diluted. Five microliter aliquot was spotted onto the LB agars containing $50 \mathrm{mg} / \mathrm{L} \mathrm{MD,} 0.01 \% \mathrm{SDS}$, and $45 \mathrm{mg} / \mathrm{L}$ DIP, respectively. Cell viabilities were recorded after 24$\mathrm{h}$ incubation at $37^{\circ} \mathrm{C}$. Each assay was independently performed at least three times and the pictures in the results was a representative.

\section{$\mathrm{H}_{2} \mathrm{O}_{2}$ sensitivity assay}

The logarithmic-phase bacterial cells of $\mathrm{OD}_{450 \mathrm{~nm}} 1.0$ were spread evenly on LB agar plates. Filter paper disks $(6 \mathrm{~mm})$ impregnated with $15 \mu \mathrm{l} 20 \% \mathrm{H}_{2} \mathrm{O}_{2}$ were placed onto the top of agar plate. After 24-h incubation at $37^{\circ} \mathrm{C}$, the growth inhibition zone was measured [19]. Each assay was independently performed at least three times.

\section{Caenorhabditis elegans survival assay}

N2 (wild type) C. elegans was acquired from Caenorhabditis Genetics Center (University of Minnesota, Minneapolis, USA). C. elegans was maintained at $20^{\circ} \mathrm{C}$ based on standard protocols [36]. The assay was carried out with 60 worms at the L4 stage and scored for survival over time. In brief, synchronized L1 larvae were obtained from gravid adults by bleach based on a previous protocol [37]. The L1 larvae were cultured on E. coli HT115 until L4 stage before transferring to $S$. maltophiliaseeded NGM agar plates supplemented with $50 \mu \mathrm{M}$ fluorodeoxyuridine (FUdR) to maintain a synchronous worm population [38]. S. maltophilia strains were grown overnight in $\mathrm{LB}$ and spread $\left(3 \times 10^{5} \mathrm{CFU} / \mathrm{cm}^{2}\right)$ onto NGM agar plates followed by overnight incubation at $37^{\circ} \mathrm{C}$. The worms exposed to S. maltophilia were scored for survival daily. During daily transfer of worms, fresh NGM agar plates seeded with sufficient bacterial food was provided regularly, which excluded the possibility that the survival of worms might be affected by the lack of food. C. elegans survival assay was analyzed by using GraphPad Prism version 8.3 (GraphPad Software, San Diego). Kaplan-Meier log rank analysis was used to determine the difference between C. elegans exposed to $S$. maltophilia strains. Each assay was independently performed at least three times.

\section{Supplementary information}

Supplementary information accompanies this paper at https://doi.org/10. 1186/s12866-020-01989-z.

Additional file 1: Table S1. Bacterial strains, plasmids, and primers used in this study.

Additional file 2: Figure. S1. SDS-PAGE and Western blotting of total bacterial proteins of S. maltophilia KJ, its derived mutants, and complementary strains. Overnight culture of bacterial cells was inoculated into fresh LB with an initial $O_{450 n m}$ of 0.15 . Cells were grown aerobically for $5 \mathrm{~h}$. Whole bacterial cell lysates were denatured and separated by $14 \%$ SDS-PAGE. (A) The gel was stained with $0.1 \%$ Coomassie Blue R250 in $10 \%$ acetic acid, $50 \%$ methanol, and $40 \% \mathrm{H}_{2} \mathrm{O}$ for $30 \mathrm{~min}$, and then destained with $10 \%$ acetic acid, $50 \%$ methanol, and $40 \% \mathrm{H}_{2} \mathrm{O}$ until the background was clear. (B) The proteins in the gel were transferred onto polyvinylidene difluoride (PVDF) membranes. The blot was reacted with anti-PhoP antibodies and secondary mouse anti-rabbit IgG immunoglobulin conjugated to horseradish peroxidase (Sigma Chemical Co., St. Louis, MO). M, protein markers; lane 1, E. coli (pETPhoP); lane 2, KJ; lane 3, KJ $\Delta$ PhoP; lane 4, KJ $\Delta$ PhoP (pPhoP); lane 5, KJ $\Delta$ PhoP (pPhoPQ); lane 6,

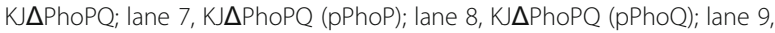
$\mathrm{K} \Delta \mathrm{PhoPQ}$ (pPhoPQ). The cropped image presented in Fig. $2 \mathrm{~b}$ is marked by a black rectangle.

Additional file 3: Figure S2. The swimming motility of wild-type $K$,

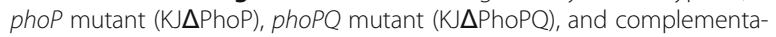
tion strain (KJPPhoPQ (pPhoPQ)). The logarithmic-phase bacterial culture was adjusted to $\mathrm{OD}_{450 \mathrm{~nm}}$ of 1.0. Five-microliter suspension was inoculated into the swimming agar ( $1 \%$ tryptone, $0.5 \% \mathrm{NaCl}$, and $0.15 \%$ agar) and the swimming zones were recorded after 48 -h incubation at $37^{\circ} \mathrm{C}$.

Additional file 4: Figure S3. The secreted protease activity of wild-type

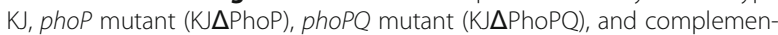
tation strain (KJPPoPQ (pPhoPQ)). The logarithmic-phase of bacterial culture was adjusted to $\mathrm{OD}_{450 \mathrm{~nm}}$ of 1.0 and $40 \mu \mathrm{l}$ bacterial aliquot were 
inoculated into the skim milk agar plate. The proteolytic activity of bacteria was assessed by measuring the transparent zones around the bacteria after incubation for $72 \mathrm{~h}$ at $37^{\circ} \mathrm{C}$.

Additional file 5: Figure S4. The expression of oxidative stress alleviation-associated genes of S. maltophilia $\mathrm{KJ}$ in response to $\mathrm{MD}$ challenge. Overnight culture of $\mathrm{KJ}$ cells was inoculated into fresh $\mathrm{LB}$ broth without or with $M D(16 \mu \mathrm{g} / \mathrm{ml})$ at an initial $\mathrm{OD}_{450 \mathrm{~nm}}$ of 0.15 . Cells were grown aerobically for $5 \mathrm{~h}$ before measuring the indicated transcripts using qRT-PCR. All values were normalized to the transcript of MD-nontreated $\mathrm{KJ}$ cells. Bars represent the average values from three independent experiments. Error bars represent the standard error of the mean. *, $P<0.001$, significance calculated by Student's $t$ test.

\section{Abbreviations}

TCS: Two-component regulatory system; SK: Sensor kinase; RR: Response regulator; CAP: Cationic antimicrobial peptides; SDS: Sodium dodecyl sulfate; DIP: 2,2'-dipyridyl; MD: Menadione; qRT-PCR: Quantitative reverse transcription-PCR

\section{Acknowledgements}

Not Applicable.

\section{Authors' contributions}

BKW, YWH, MZL, MFL, and HJH carried out the experiments. HFL, HCY, and TCY designed the experiments, analyzed the data, and wrote the manuscript. All authors have read and approved the manuscript.

\section{Funding}

This work was supported by the Ministry of Science and Technology of Taiwan [grant numbers MOST 108-2320-B-010-032-MY3 and MOST 1082320-B-264-002], the Cheng Hsin General Hospital [grant numbers CY10928 and CHGH109-37], and the Professor Tsuei-Chu Mong Merit Scholarship (grant no. 30719003). The funding bodies played no role in the design of the study and collection, analysis, and interpretation of data and in writing the manuscript.

\section{Availability of data and materials}

The datasets used and/or analysed during the current study available from the corresponding author on reasonable request.

\section{Ethics approval and consent to participate}

Not applicable.

\section{Consent for publication}

Not applicable.

\section{Competing interests}

The authors declare that they have no conflict of interest.

\section{Author details}

'Department of Clinical Pathology, Cheng Hsin General Hospital, Taipei, Taiwan. '2Department of Restaurant, Hotel and Institutional Management, Fu-Jen Catholic University, New Taipei City, Taiwan. ${ }^{3}$ Department of Biotechnology and Laboratory Science in Medicine, National Yang-Ming University, Taipei, Taiwan. ${ }^{4}$ Department of Medical Laboratory Science and Biotechnology, Yuanpei University of Medical Technology, Hsinchu, Taiwan.

Received: 19 May 2020 Accepted: 30 September 2020

Published online: 14 October 2020

\section{References}

1. Gooderham WJ, Hancock RE. Regulation of virulence and antibiotic resistance by two-component regulatory systems in Pseudomonas aeruginosa. FEMS Microbiol Rev. 2009;33:279-94.

2. Singh K, Senadheera DB, Cvitkovitch DG. An intimate link: two-component signal transduction systems and metal transport systems in bacteria. Future Microbiol. 2014;9:1283-93.

3. Groisman EA. The pleiotropic two-component regulatory system PhoPPhoQ. J Bacteriol. 2001;183:1835-42.
4. Garcia Vescovi E, Soncini FC, Groisman EA. $\mathrm{Mg}^{2+}$ as an extracellular signal: environmental regulation of Salmonella virulence. Cell. 1996;84:165-74.

5. Bader MW, Sanowar S, Daley ME, Schneider AR, Cho U, Xu W, Klevit RE, Le Moual H, Miller SI. Recognition of antimicrobial peptides by a bacterial sensor kinase. Cell. 2005;122:461-72.

6. Prost LR, Daley ME, Le Sage V, Bader MW, Le Moual H, Klevit RE, Miller SI. Activation of the bacterial sensor kinase PhoQ by acidic pH. Mol Cell. 2007; 26:165-74.

7. Yuana J, Jina F, Glattera T, Sourjik V. Osmosensing by the bacterial PhoQ/ PhoP two-component system. Proc Natl Acad Sci U S A. 2007;28:E10793.

8. Kato A, Groisman EA. The PhoQ/PhoP regulatory network of Salmonella enterica. Adv Exp Med Biol. 2008;631:7-21.

9. Li Y, Gao H, Qin L, Li B, Han Y, Guo Z, Song Y, Zhai J, Du Z, Wang X, Zhou $D$, Yang R. Identification and characterization of PhoP regulon members in Yersinia pestis biovar Microtus. BMC Genomics. 2008;9:143.

10. Kato A, Tanabe H, Utsumi R. Molecular characterization of the PhoP-PhoQ two-component system in Escherichia coli K-12: identification of extracellular $\mathrm{Mg}^{2+}$-responsive promoters. J Bacteriol. 1999;181:5516-20.

11. Gooderham WJ, Gellatly SL, Sanschagrin F, McPhee JB, Bains M, Cosseau C, Levesque RC, Hancock RE. The sensor kinase PhoQ mediates virulence in Pseudomonas aeruginosa. Microbiology. 2009;155:699-711.

12. Liu MC, Tsai YL, Huang YW, Chen HY, Hsueh PR, Lai SY, Chen LC, Chou YH, Lin WY, Liaw SJ. Stenotrophomonas maltophilia PhoP, a two-component response regulator, involved in antimicrobial susceptibilities. PLoS One. 2016;11:e0153753.

13. Brooke JS. Stenotrophomonas maltophilia: an emerging global opportunistic pathogen. Clin Microbiol Rev. 2012;25:2-41.

14. Chang YT, Lin CY, Chen YH, Hsueh PR. Update on infections caused by Stenotrophomonas maltophilia with particular attention to resistance mechanisms and therapeutic options. Front Microbiol. 2015;6:893.

15. Crossman LC, Gould VC, Dow JM, Vernikos GS, Okazaki AK, Sebaihia M, Saunders D, Arrowsmith C, Carver T, Peters N, Adlem E, Kerhornou A, Lord A, Murphy L, Seeger K, Squares R, Rutter S, Quail MA, Rajandrea MA, Harris D, Churcher C, Bentley SD, Parkhill J, Thomson NR, Avison MB. The complete genome, comparative and functional analysis of Stenotrophomonas maltophilia reveals an organism heavily shielded by drug resistance determinants. Genome Biol. 2008;9:R74.

16. Jair HW, Lu HF, Huang YW, Pan SY, Lin IL, Huang HH, Yang TC. Roles of the two-MnSOD system of Stenotrophomonas maltophilia in the alleviation of superoxide stress. Int J Mol Sci. 2019:20:1770.

17. Li LH, Shih YL, Huang JY, Wu CJ, Huang YW, Huang HH, Tsai YC, Yang TC. Protection from hydrogen peroxide stress relies mainly on AhpCF and KatA2 in Stenotrophomonas maltophilia. J Biomed Sci. 2020;27:37.

18. Lin YT, Huang YW, Liou RS, Chang YC, Yang TC. MacABCsm, an ABC-type tripartite efflux pump of Stenotrophomonas maltophilia involved in drug resistance, oxidative and envelope stress tolerances and biofilm formation. J Antimicrob Chemother. 2014;69(12):3221-6.

19. Lin YT, Huang YW, Chen SJ, Chang CW, Yang TC. The SmeYZ efflux pump of Stenotrophomonas maltophilia contributes to drug resistance, virulencerelated characteristics, and virulence in mice. Antimicrob Agents Chemother. 2015:59:4067-73.

20. Wu CJ, Chiu TT, Lin YT, Huang YW, Li LH, Yang TC. Role of smeU1WWU2X operon in alleviation of oxidative stresses and occurrence of sulfamethoxazole-trimethoprim-resistant mutants in Stenotrophomonas maltophilia. Antimicrob Agents Chemother. 2018:62:e02114-7.

21. Li LH, Wu CM, Lin YT, Pan SY, Yang TC. Roles of FadRACB system in formaldehyde detoxification, oxidative stress alleviation and antibiotic susceptibility in Stenotrophomonas maltophilia. J Antimicrob Chemother 2020;75:2101-9.

22. Peng BY, Pan Y, Li RJ, Wei JW, Liang F, Wang L, Wang FF, Qian W. An essential regulatory system originating from polygenic transcriptional rewiring of PhoP-PhoQ of Xanthomonas campestris. Genetics. 2017;206: 2207-23.

23. Macfarlane EL, Kwasnicka A, Ochs MM, Hancock RE. PhoP-PhoQ homologues in Pseudomonas aeruginosa regulate expression of the outermembrane protein OprH and polymyxin B resistance. Mol Microbiol. 1999; 34:305-16.

24. Yan $\mathrm{Q}$, Wu XG, Wei HL, Wang HM, Zhang LQ. Differential control of the Pcol/PcoR quorum-sensing system in Pseudomonas fluorescens 2P24 by sigma factor RpoS and the GacS/GacA two-component regulatory system. Microbiol Res. 2009;164:18-26. 
25. Chen L, Duan K. A PhoPQ-regulated ABC transporter system exports tetracycline in Pseudomonas aeruginosa. Antimicrob Agents Chemother. 2016;60:3016-24.

26. Mishra S, Imlay J. Why do bacteria use so many enzymes to scavenge hydrogen peroxide? Arch Biochem Biophys. 2012;525:145-60.

27. Poole K. Stress responses as determinants of antimicrobial resistance in Pseudomonas aeruginosa: multidrug efflux and more. Can J Microbiol. 2014; 60:783-91.

28. Demple B. Regulation of bacterial oxidative stress genes. Annu Rev Genet. 1991;25:315-37.

29. Kato A, Tanabe H, Utsumi R. Molecular characterization of the PhoP-PhoQ two-component system in Escherichia coli K12: identification of extracellular $\mathrm{Mg}^{2+}$ responsive promoters. J Bacteriol. 1999;181:5516-20.

30. McPhee JB, Bains M, Winsor G, Lewenza S, Kwasnicka A, Brazas MD, Brinkman FSL, Hancock REW. Contribution of the PhoP-PhoQ and PmrAPmrB two-component regulatory systems to $\mathrm{Mg}^{2+}$-induced gene regulation in Pseudomonas aeruginosa. J Bacteriol. 2006:188:3995-4006.

31. Frees $D$, Brondsted $L$, Ingmer H. Bacterial proteases and virulence. Subcell Biochem. 2013;66:161-92.

32. Huang YW, Huang HH, Huang KH, Chen WC, Lin YT, Hsu CC, Yang TC. Ampl functions as an iron exporter to alleviate $\beta$-lactam-mediated reactive oxygen species stress in Stenotrophomonas maltophilia. Antimicrob Agents Chemother. 2019;63:e02467-18.

33. Yang TC, Huang YW, Hu RM, Huang SC, Lin YT. AmpD, is involved in expression of the chromosomal L1 and L2 beta-lactamases of Stenotrophomonas maltophilia. Antimicrob Agents Chemother. 2009:53:2902-7.

34. Livak KJ, Schmittgen TD. Analysis of relative gene expression data using real-time quantitative PCR and the 2-(delta deltaC(T)) method. Methods. 2001;25:402-8

35. CLSI, editor. Performance standards for antimicrobial susceptibility testing 29th ed. CLSI supplement M100. Wayne: Clinical and Laboratory Standards Institute; 2019.

36. Girard LR, Fiedler TJ, Harris TW, Carvalho F, Antoshechkin I, Han M, Sternberg PW, Stein LD, Chalfie M. WormBook: the online review of Caenorhabditis elegans biology. Nucleic Acids Res. 2007;35:D472-5.

37. Yang WH, Chen CY, Wang KL, Kwok HL, Stern A, Lo SJ, Yang HC. Reflex and habituation behavior of Caenorhabditis elegans assessed by a mechanical vibration system and image analysis. J Neurosci Methods. 2019;328:108415.

38. Mitchell DH, Stiles JW, Santelli J, Sanadi DR. Synchronous growth and aging of Caenorhabditis elegans in the presence of fluorodeoxyuridine. J Gerontol. 1979;34:28-36.

\section{Publisher's Note}

Springer Nature remains neutral with regard to jurisdictional claims in published maps and institutional affiliations.

Ready to submit your research? Choose BMC and benefit from:

- fast, convenient online submission

- thorough peer review by experienced researchers in your field

- rapid publication on acceptance

- support for research data, including large and complex data types

- gold Open Access which fosters wider collaboration and increased citations

- maximum visibility for your research: over $100 \mathrm{M}$ website views per year

At $\mathrm{BMC}$, research is always in progress.

Learn more biomedcentral.com/submissions 